\title{
Editorial
}

\section{Hotspots in Psychology - 2022 Edition}

\author{
Holger Steinmetz ${ }^{1}$, Nadine Wedderhoff ${ }^{1}$, and Michael Bošnjak ${ }^{1,2}$ \\ ${ }^{1}$ University of Trier, Germany \\ ${ }^{2}$ Department of Epidemiology and Health Monitoring, Robert Koch Institute, Berlin, Germany
}

\begin{abstract}
This editorial introduces the five articles included in the sixth "Hotspots in Psychology" of the Zeitschrift für Psychologie. With the new edition, the format enlarges it focus beyond meta-analyses and systematic reviews to new developments such as big data in psychology. The included five articles span a diverse array of topics, that is, the application of individual participants meta-analyses as a way to replicate studies, the role of the degree of anthropomorphism ("human-likeness") in human-robot interactions, the challenge of multiple dependent effect sizes when conducting a meta-analytical structural equation model, the value of using log data of online platform as a way to predict learning outcomes, and the utility of a block-wise fit evaluation in structural equation models with many longitudinally measured variables. To promote the open science philosophy, the papers present supplemental material that can be accessed via the repository PsychArchives (www. psycharchives.org)
\end{abstract}

The "Hotspots in Psychology" topical issue has a tradition in presenting articles focusing on reviews and meta-analyses in research-active (i.e., hotspot) fields (Bošnjak \& Erdfelder, 2018; Bošnjak \& Gnambs, 2019; Bošnjak \& Wedderhoff, 2020; Bošnjak, Wedderhoff, \& Steinmetz 2021; Erdfelder \& Bošnjak, 2016). While the focus on metaanalyses is still the backbone of this year's edition, we decided to widen the focus to also include articles discussing the fruitfulness and challenges of big data, for instance, of intensive longitudinal data (e.g., time series, experience sampling), data provided by means of nonobtrusive methods (e.g., sensors, log data), and approaches related to the treatment and handling of such data or their statistical analysis.

The first article by Maria Klose, Diana Steger, Julian Fick, and Cordula Artelt (2022) focuses on the utility and challenges of analyzing log data on learning activities in the field of learning analytics. The authors present a metaanalysis using 41 studies $(N=28,986)$, revealing a positive relationship between total login time and login frequency on a learning platform and grades. Beyond the specific implications of the usefulness of log data for analyzing learning behavior and outcomes, the paper fits in with the increasing research on the value of big, technically measured data for psychology and related fields.

The article by Isidora Stolwijk, Suzanne Jak, Veroni Eichelsheim, and Machteld Hoeve (2022) addresses a challenge most researchers testing meta-analytical structural equation models (MASEM) are confronted with: how to proceed when primary studies present several estimates of a target correlation. While this problem can easily be handled through a three-level approach when the target is a simple bivariate correlation, issues become tricky when the goal is a MASEM based on a matrix of correlations. Stolwijk and colleagues compare the differences among several approaches (i.e., ignoring, aggregation, elimination, and applying a multilevel approach). Based on the severe differences among the analytical approaches, the authors recommend relying on the multilevel approach as the approach that fully considers the nested structure of the data.

The paper by Martina Mara, Markus Appel, and Timo Gnambs (2022) reports a meta-analysis in the field of human-robot interaction, especially on the Uncanny Valley Hypothesis, which claims that there is a curvilinear relation-ship between the evaluation of a robot as likable and the extent of anthropomorphism (i.e., the creation of the robot as human-like). According to the hypothesis, individuals will increasingly react with positive feelings to robots with their increasing human-likeness but - beyond a certain point - will tend to negatively react to even increasing likeness. At the extreme value of humanlikeness, however, reactions will turn positive again. Using data from $49(N=3,556)$ studies and focusing of studies that used the most widely used instruments (i.e., the Godspeed scales), the polynomial meta-regression analysis shows a nonlinear but monotonic relationship between anthropomorphism and likeability. While these results could be interpreted as counterevidence to the uncanny valley hypothesis, the authors conclude that the limited amount of data addressing the extreme part of the anthropomorphism dimension is the most direct conclusion.

The article by Julia Norget and Axel Mayer (2022) addresses the question of how to evaluate the fit of structural equation model applied to longitudinal data, for instance, from experience sampling designs. Based on their 
argument that common fit indices perform poorly in models estimated with many variables measured in experience sampling, the authors propose and analyze the value of a block-wise fit assessment for such models. The authors present two simulation models that support the value of their approach but also shows some limitation such that some misspecified models cannot be detected and that the assessment of fit naturally is focused and, thus, limited on misspecifications in the respective block.

In the fifth and final article, Robbie van Aert (2022) discusses the value and benefits of conducting an individual participants meta-analysis when analyzing multiple replications of studies conducted in different labs that have become prominent in the many labs project (Ebersole et al., 2016). He points out to the weaknesses of meta-analytical approaches to aggregate the results of these studies, most notably the lower statistical power when analyzing moderator effects (in the form of differences across studies) and - more importantly - the danger of aggregation biases, that is, falsely concluding differences between studies to differences between individuals. As a remedy, he provides an introduction and tutorial with $\mathrm{R}$ to individual participant meta-analysis, in which the primary data of all studies are modeled in a multilevel model in which differences across labs can be represented in the form of random effects.

Overall, we believe that the broadened methodological scope has contributed to presenting an illustration of a broad array of topics highlighting developments and emerging themes in psychology.

\section{References}

Bošnjak, M., \& Erdfelder, E. (2018). Hotspots in Psychology - 2018 Edition. Zeitschrift für Psychologie, 226(1), 1-2. https://doi.org/ 10.1027/2151-2604/a000323

Bošnjak, M., \& Gnambs, T. (2019). Hotspots in Psychology - 2019 Edition. Zeitschrift für Psychologie, 227(1), 1-3. https://doi.org/ 10.1027/2151-2604/a000350

Bošnjak, M., \& Wedderhoff, N. (2020). Hotspots in Psychology 2020 Edition. Zeitschrift für Psychologie, 228(1), 1-2. https://doi.org/10.1027/2151-2604/a000398
Bošnjak, M., Wedderhoff, N., \& Steinmetz, H. (2021). Hotspots in Psychology - 2021 Edition. Zeitschrift für Psychologie, 229(1), 1-2. https://doi.org/10.1027/2151-2604/a000438

Ebersole, C. R., Atherton, O. E., Belanger, A. L., Skulborstad, H. M., Allen, J. M., Banks, J. B., Baranski, E., Bernstein, M. J., Bonfiglio, D. B. V., Boucher, L., Brown, E. R., Budiman, N. I., Cairo, A. H., Capaldi, C. A., Chartier, C. R., Chung, J. M., Cicero, D. C., Coleman, J. A., Conway, J. G., ... Nosek, B. A. (2016). Many labs 3: Evaluating participant pool quality across the academic semester via replication. Journal of Experimental Social Psychology, 67, Article 68015082. https://doi.org/ 10.1016/j.jesp.2015.10.012

Erdfelder, E., \& Bošnjak, M. (2016). Hotspots in Psychology: A new format for special issues of the Zeitschrift für Psychologie. Zeitschrift für Psychologie, 224(3), 141-144. https://doi.org/ 10.1027/2151-2604/a000249

Klose, M., Steger, D., Fick, J., \& Artelt, C. (2022). Decrypting log data: A meta-analysis on general online activity and learning outcome within digital learning environments. Zeitschrift für Psychologie, 230(1), 3-15. https://doi.org/10.1027/2151-2604/ a000484

Mara, M., Appel, M., \& Gnambs, T. (2022). Human-like robots and the uncanny valley: A meta-analysis of user responses based on the Godspeed scales. Zeitschrift für Psychologie, 230(1), 3346. https://doi.org/10.1027/2151-2604/a000486

Norget, J., \& Mayer, A. (2022). Block-wise model fit for structural equation models with experience sampling data. Zeitschrift für Psychologie, 230(1), 47-59. https://doi.org/10.1027/21512604/a000482

Stolwijk, I., Jak, S., Eichelsheim, V., \& Hoeve, M. (2022). Dealing with dependent effect sizes in MASEM: A comparison of different approaches using empirical data. Zeitschrift für Psychologie, 230(1), 16-32. https://doi.org/10.1027/21512604/a000485

Van Aert, R. C. M. (2022). Analyzing data of a multilab replication project with individual participant data meta-analysis: A tutorial. Zeitschrift für Psychologie, 230(1), 60-72. https://doi. org/10.1027/2151-2604/a000483

Published online February 2, 2022

\section{Holger Steinmetz}

Faculty of Management

University of Trier

Universitätsring 15

54296 Trier

Germany

steinmetzh@uni-trier.de 